\title{
Efficacy of video-assisted anal fistula treatment combined with closure of the internal opening using a stapler for Parks II anal fistula
}

\author{
Yuru Zhang ${ }^{1,2}$, Fei $\mathrm{Li}^{1}$, Tuanjie Zhao ${ }^{2}$, Feng Cao ${ }^{1}$, Yamin Zheng ${ }^{1}$, Ang $\mathrm{Li}^{1}$ \\ ${ }^{1}$ Department of General Surgery, Xuanwu Hospital, Capital Medical University, Beijing, China; ${ }^{2}$ Department of Colorectal Surgery, Beijing Erlonglu \\ Hospital, Beijing, China \\ Contributions: (I) Conception and design: Y Zhang, F Li (II) Administrative support: F Li; (III) Provision of study materials or patients: T Zhao, A Li; \\ (IV) Collection and assembly of data: F Cao; (V) Data analysis and interpretation: Y Zheng; (VI) Manuscript writing: All authors; (VII) Final approval \\ of manuscript: All authors. \\ Correspondence to: Fei Li. Department of General Surgery, Xuanwu Hospital, Capital Medical University, No.45 Changchun Street, Xicheng District, \\ Beijing 100053, China. Email: feili36@ccmu.edu.cn.
}

\begin{abstract}
Background: To explore the efficacy of video-assisted anal fistula treatment (VAAFT) combined with an internal-opening closure technique using a stapler in the treatment of Parks II anal fistula.

Methods: From September 2017 to June 2019, 75 patients with Parks II anal fistulas in Beijing Erlonglu Hospital were enrolled and randomly allocated into two groups. The 37 patients in the treatment group received VAAFT combined with internal-opening closure techniques, and the 38 patients in the control group were treated with anal fistulotomy with seton placement. The primary outcomes included the healing rate and recurrence, the fecal incontinence severity index (FISI) score, and the Wexner incontinence score during the 6-month postoperative follow-up.

Results: Thirty-two cases were healed in the treatment group (86.5\%) and thirty-six cases were cured in the control group (94.7\%). There was no notable difference in the healing rate between the two groups $(\mathrm{P}=0.487)$. Significant differences between the groups were observed in the Wexner incontinence and FISI scores at 1,3 , and 6 months after the operation $(\mathrm{P}=0.001)$. Furthermore, the wound healing time in the treatment group was significantly shorter than in the control group $(\mathrm{P}<0.05)$, while the numerical rating scale (NRS) for postoperative pain on the first day and 1 week after the operation were significantly lower in the treatment group than in the control group $(\mathrm{P}<0.05)$.

Conclusions: VAAFT combined with closure of the internal opening using a stapler is effective and safe for Parks II anal fistula, and should be promoted as a promising treatment.
\end{abstract}

Keywords: Video-assisted anal fistula treatment (VAAFT); stapler; internal opening; Parks II; anal function

Submitted Sep 23, 2020. Accepted for publication Nov 13, 2020.

doi: $10.21037 /$ atm-20-7154

View this article at: http://dx.doi.org/10.21037/atm-20-7154

\section{Introduction}

The treatment of a complex anal fistula is a considerable challenge for colorectal surgeons, as traditional fistulotomy with seton placement can lead to a high anal incontinence rate (1). In recent years, many attempts have been made to preserve anal function and reduce the incidence of postoperative anal incontinence. Meinero et al. reported on a video-assisted anal fistula treatment (VAAFT) for the first time (2), and since the introduction of this technique in 2015, positive outcomes have been achieved in the treatment of complex anal fistulas in our hospital. By reviewing the clinical data of patients with Parks II anal fistulas between 2017 and 2019 in this study, we evaluated the efficacy of VAAFT and aimed to provide a reference for 


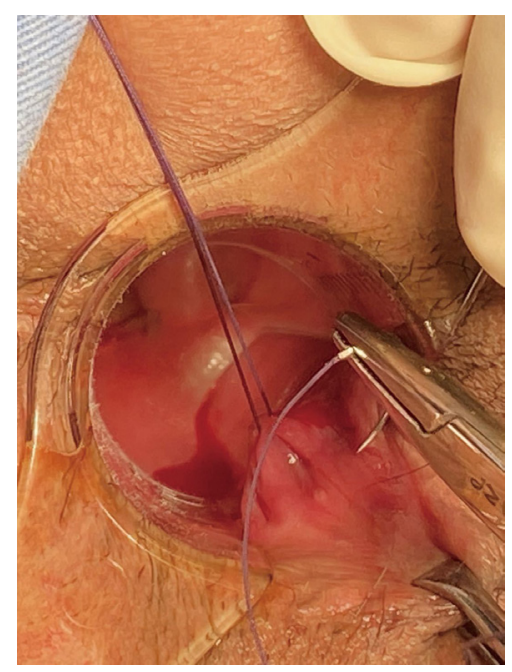

Figure 1 Location of internal opening. Membranes on the upper and lower sides of the internal opening were sutured and lifted.

the selection of surgical methods for complex anal fistula treatment.

We present the following article in accordance with the CONSORT reporting checklist (available at http://dx.doi. org/10.21037/atm-20-7154).

\section{Methods}

\section{Patients}

From September 2017 to June 2019, a total of 75 patients with Parks II anal fistulas were selected and randomized into treatment and control groups. VAAFT was administered to 37 patients (treatment group) and anal fistulotomy with seton placement was performed on the remaining 38 patients (control group). There were no significant differences between the patients' ages and genders $(\mathrm{P}>0.05)$. The patients, aged $18-65$ years, were preoperatively diagnosed with Parks II anal fistula via threedimensional ultrasound or magnetic resonance imaging (MRI). Their anal functions were normal, without laxity, stenosis, infection, or malformation; their physical assessment results were unremarkable; and cardiopulmonary function evaluation showed they were low risk for surgery. Patients with inflammatory bowel disease, malignant tumors, severe organ dysfunction, or severe diabetes were excluded from our study.

Written informed consent was obtained from all patients prior to surgery. The study protocol was conducted in accordance with the Declaration of Helsinki (as revised in 2013), and approved by the Research Ethics Committee of our hospital (2019ELLHA-001-01). The trial was registered at the Chinese Clinical Trial Registry (ChiCTR1900022810), and the work has been reported in line with the strengthening the reporting of cohort studies in surgery (STROCSS) criteria (3).

\section{Surgical procedure}

\section{Treatment group}

Spinal anesthesia was administered to each patient. During positioning, we kept the fistula at a lower position in the body for easier maneuvering, according to the location of the fistula (i.e., patients with left-sided anal fistulas were positioned in the left lateral position, patients with rightsided anal fistula were placed in the right lateral position, patients with posterior anal fistulas were positioned in lithotomy, and patients with anterior anal fistulas were placed in the jack-knife position). Next, we inserted the fistuloscope through the external opening and cautiously monitored its movement on the screen. The fistuloscope was gently and slowly advanced into the fistula to further straighten it. Meanwhile, continuous jetting of solution was carried out to ensure optimal visualization of the fistula lumen until the internal opening was localized and marked with 2-3 stitches at the edge. Subsequently, the filler was removed and replaced with the electrode, which destroyed the fistula tract wall under direct vision to cauterize all fragments of the whitish material attached to the fistula tract wall, covering all abscess cavities and fistula tract. The external fistula opening was enlarged and resected. We then returned to the rectum to close the internal opening using a stapler and ultimately performed hemostasis. Furthermore, drainage or negative pressure suction was applied to the external opening. The VAAFT surgical procedure is shown in Figures 1-3.

\section{Control group}

Spinal anesthesia was also required for patients in the control group. Patient positioning was the same as in the treatment group. A probe was gently inserted into the fistula tract through the external opening, and the fistula tract was incised while probing until the internal opening was reached. Meanwhile, the fistula branches were also opened while probing, if applicable. To protect anal sphincter function during the operation, we placed the seton in the external anal sphincter below the fistula, a portion of the 


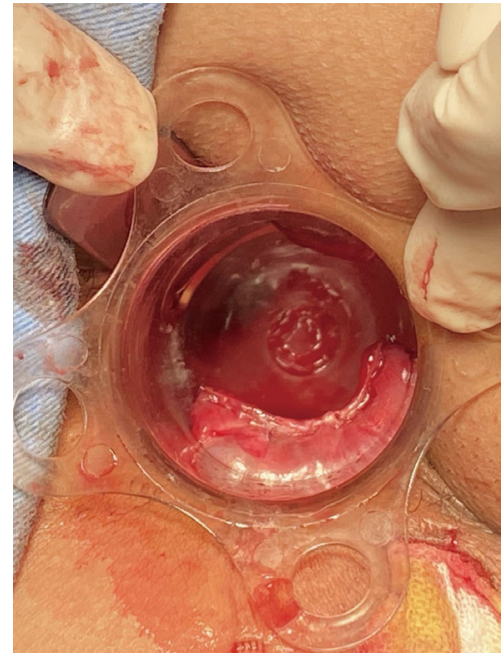

Figure 2 Closure of the internal opening. The internal opening was closed horizontally with a staple.

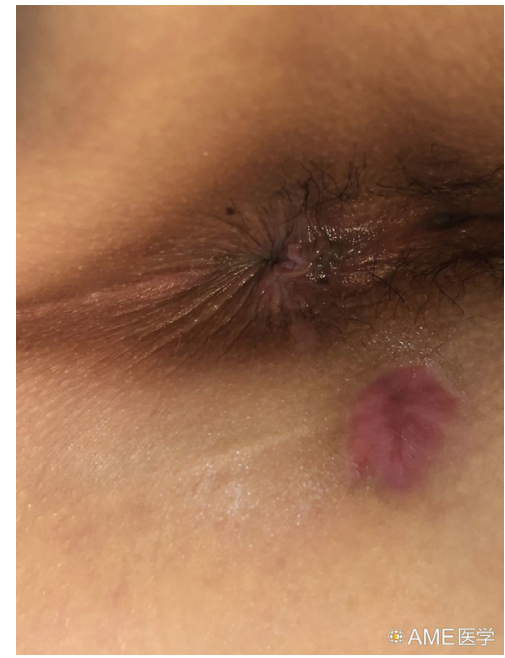

Figure 3 Wound healing. Wound healing status 1 month after surgery.

internal anal sphincter, and the internal opening of the fistula. The fistula was cleared of necrotic granulation tissue and the wound was trimmed to create a V-shaped wound with a small base and large opening to facilitate wound drainage and healing.

\section{Outcomes}

\section{Primary outcomes}

The healing rate and recurrence, fecal incontinence severity index (FISI) score, and Wexner incontinence score during the 6-month postoperative follow-up.

\section{Secondary outcomes}

The operating time, intraoperative blood loss, urinary retention, edema of the wound, postoperative hospital stays, wound healing time, and numerical rating scale (NRS) for pain.

\section{Statistical analysis}

SPSS 20.0 for Windows (IBM Corp., Armonk, NY, USA) was used to analyze the data. Specifically, the measurement data were described by means \pm standard deviation, median, maximum and minimum. The enumeration data and ranked data were described as the number of samples and percentages. A paired $t$-test or nonparametric test was used to compare with baseline values for measurement data, and a rank-sum test was used for enumeration data. A P value of $<0.05$ was considered statistically significant.

\section{Results}

In this study, a total of 75 subjects with Parks II anal fistula were screened and enrolled in accordance with the inclusion and exclusion criteria, including 37 patients in the treatment group (male: 28, female: 9 ) and 38 patients in the control group (male: 32 , female: 6). No significant difference in gender was found between the two groups $(\mathrm{P}=0.356)$. Similarly, there was no significant difference in patients' ages between the two groups $(\mathrm{P}=0.969)$, which were $36.70 \pm 9.24$ in the treatment group and $36.79 \pm 10.05$ in the control group. The average operating time for the treatment group was 49 minutes, which was approximately 15 minutes longer than that for the control group, showing a notable difference between the two groups. There was also a statistically significant difference in intraoperative bleeding between the two groups $(\mathrm{P}<0.05)$, with less bleeding in the treatment group compared with the control group. The surgical outcomes are shown in Table 1.

Comparison of surgical outcomes between the VAAFT and control groups are shown in Table 2. There were no notable differences in the incidence of postoperative complications, such as urinary retention, edema of the wound, and bleeding between the two groups $(\mathrm{P}>0.05)$. In addition, there was no significant difference in postoperative hospital stays between the groups $(\mathrm{P}>0.05)$. The NRS was employed to assess pain on the first day, 1 
Table 1 Patients characteristics

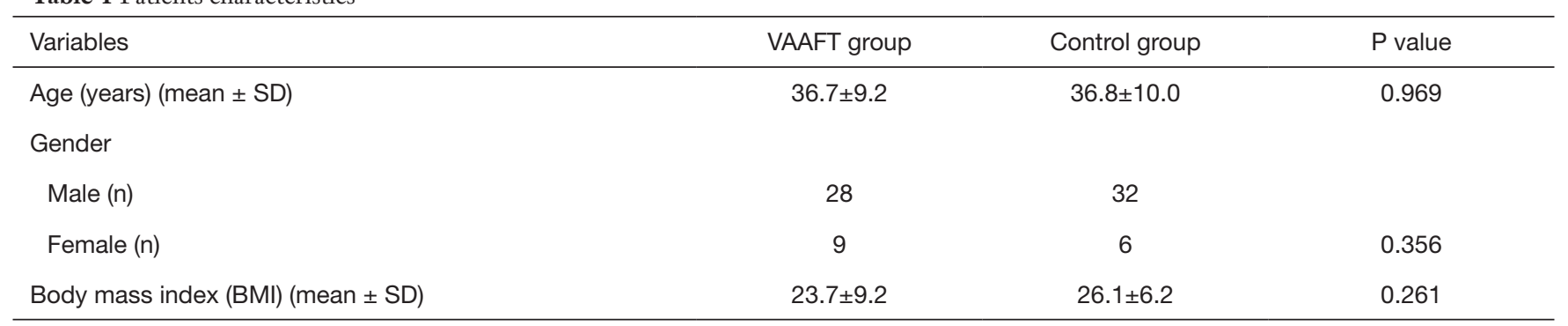

VAAFT, video-assisted anal fistula treatment; SD, standard deviation.

Table 2 Comparison of surgical outcomes between the VAAFT and control groups

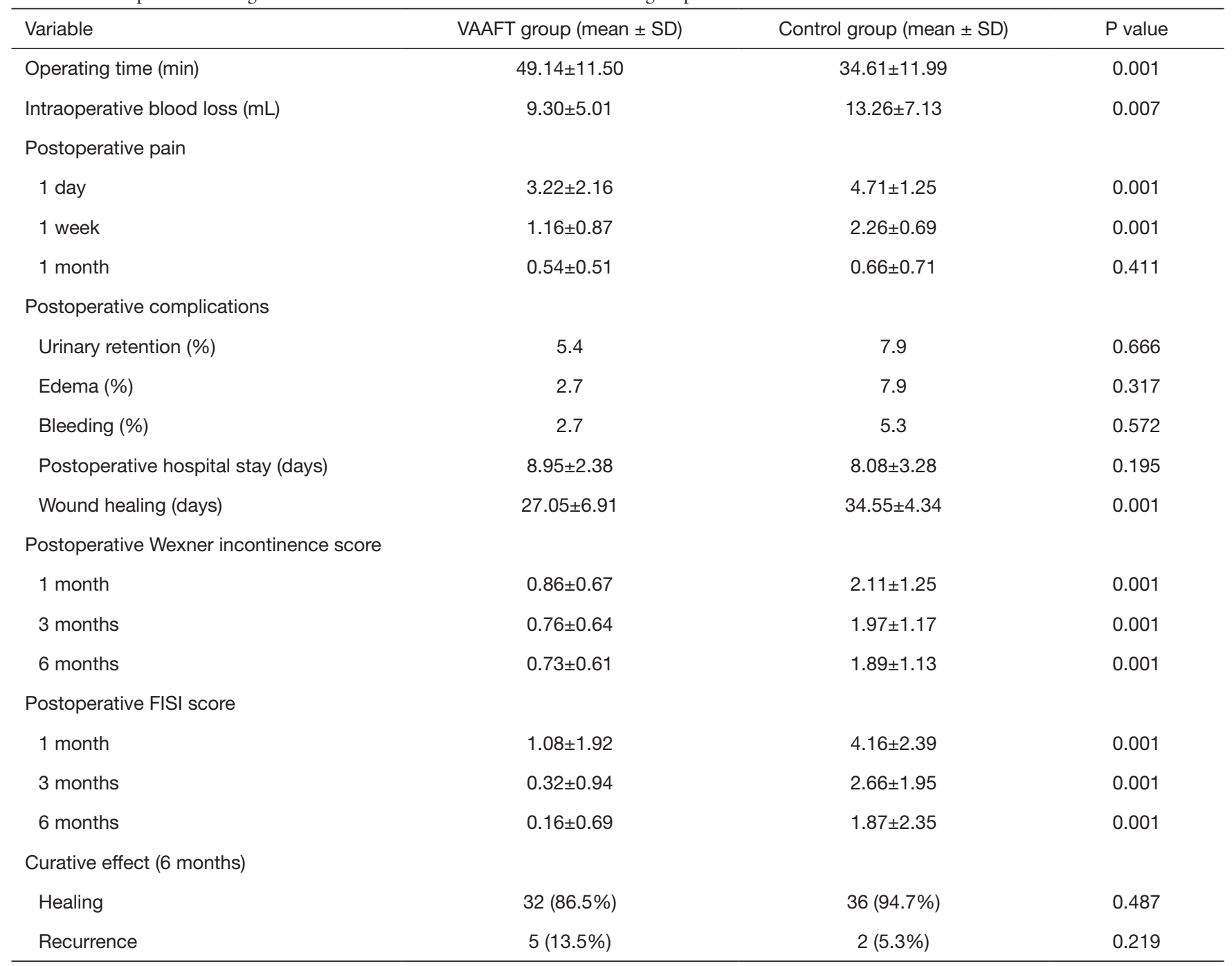

VAAFT, video-assisted anal fistula treatment; SD, standard deviation; FISI, fecal incontinence severity index. 
week, and 1 month following surgery. Compared with the control group, the NRS results of the treatment group were considerably lower on the first day and 1 week after the operation $(\mathrm{P}=0.001)$, yet no significant difference was found between the two groups at 1 month after surgery $(\mathrm{P}=0.411)$. Also, patients in the treatment group went through a shorter wound healing duration (with a mean of 27 days) compared with the control group, and the difference in the wound healing time between the two groups was statistically significant $(\mathrm{P}<0.05)$. Moreover, a marked difference was also found in the Wexner incontinence and FISI scores at 1 month, 3 months, and 6 months postoperatively $(\mathrm{P}=0.001)$.

Six months after the operation, all patients were followed up by telephone or hospital visits to identify fistula recurrence by assessing symptoms, such as perianal swelling and pain or the presence of an abscess with discharge. Fistula recurrence was found in five patients in the treatment group and in two patients in the control group, and the recurrence rates were $13.5 \%(5 / 37)$ and $5.3 \%(2 / 38)$ in the treatment and control groups, respectively. However, the difference between the two groups was not statistically significant $(\mathrm{P}=0.219)$. All patients with fistula recurrence were ultimately cured by additional anal fistulotomy with seton placement.

\section{Discussion}

To date, surgery for the treatment of anal fistula remains difficult and intricate, as improper treatment can lead to anal malfunctions, partial anal incontinence, and even complete anal incontinence. Therefore, it is imperative to balance the risks of fecal incontinence and postoperative recurrence when selecting the surgical technique for treatment of complex anal fistula (4). Although fistulectomy and fistulotomy are common surgeries for fistulas and can achieve a high healing rate (5), they are associated with a high risk of anal dysfunction and partial anal incontinence $(1,6)$. Therefore, treatment must be carefully planned based on the type of anal fistula.

Better protecting the anal sphincter while reducing the recurrence rate of anal fistulas and avoiding sphincter impairment is an intractable issue that needs to be addressed by colorectal surgeons. Efforts have been made to protect patients' anal sphincter function and avoid the occurrence of postoperative fecal incontinence by developing techniques such as VAAFT (2), anal fistula plug [AFP (7), ligation of inter sphincteric fistula tract (LIFT) (8)], fistula laser closure [FiLaC (9), and autologous stem cell transplantation (10)].
Meinero $\mathrm{et} \mathrm{al}$. reported the effectiveness of VAAFT in 136 patients with complex anal fistulas. In their report, 72 of the 136 patients $(73.5 \%)$ achieved primary healing within 2-3 months after the operation; 98 of the 136 patients completed a 6-month follow-up, and there were no severe complications during this period; 62 subjects were followed up for more than 1 year and $87.1 \%$ achieved primary healing (2). These favorable outcomes were achieved because the anal sphincter was preserved in VAAFT. During the operation, a fistuloscope was deployed as part of the anal fistula treatment without employing fistulectomy or fistulotomy processes, thereby eliminating the risks of anal sphincter impairment. Importantly, the principal considerations for treating anal fistulas involve accurate identification of the fistula tract and internal opening, resectioning the fistula, and protecting anal sphincter function. The VAFFT technique ideally addresses these three considerations by precisely locating the fistula and closing the internal opening, damaging the anal fistula tract and its branches, and cleaning up necrotic tissues in the fistula tract, thus improving wound healing $(2,11)$.

The VAAFT approach has been extensively studied and was initially applied to anal fistula treatment (12-15). It is considered to be a potentially effective alternative to conventional anal fistula surgery because of the anal sphincter-saving benefits it offers $(14,16,17)$, even in children $(18,19)$ and patients with Crohn's disease $(20,21)$. Zarin et al. applied VAAFT in 40 patients with anal fistulas; the operation failed in three patients, while the remaining 37 patients were followed up for 6 weeks to 6 months: $50 \%$ of the 40 patients achieved primary healing and $42.5 \%$ achieved secondary healing (22). Hence, the authors concluded that not only can VAAFT correctly locate the internal opening, remove the fistula, and preserve the function of the anal sphincter, but also improve patient satisfaction and reduce surgical scarring (22). Emile et al. conducted a meta-analysis on 788 anal fistula patients, and their results showed that the internal openings were located in $85.7 \%$ of patients, the mean operating time was $42 \pm 14.2$ minutes, the median follow-up duration was 9 months, and the recurrence rate was $14.2 \%$. Furthermore, the recurrence rate was related to the closure techniques of the internal openings. In particular, closure using a stapler is associated with a lower recurrence rate than by using the rectal advancement flap (23). Adegbola et al. reviewed 12 VAAFT clinical studies and concluded that VAAFT is a safe and effective option for the treatment of anal fistulas with high healing rates in the short term without causing 
anal incontinence (12). Although the preliminary data for VAAFT exhibits encouraging outcomes, Amato et al. pointed out that more multi-centered, randomized control trials are needed to further validate the efficacy of VAAFT for complex anal fistula treatment (24).

In our study, we found five cases of recurrence in the treatment group with a recurrence rate of $13.5 \%$, and two cases of recurrence in the control group with a recurrence rate of $5.3 \%$ after 6 months. Although there were no significant differences in recurrence or healing rates between the two groups, the healing rate in the control group was higher than in the treatment group. The reason for this discrepancy may be explained by the following factors: traditional anal fistulotomy with seton placement is a well-developed surgical procedure (5); the anatomical structure of the anal fistula is clearly visible, the necrotic tissues in the fistula tract are removed, and the surgeons are more familiar with this technique and can perform better wound management. Meanwhile, VAAFT is a novel technique that was introduced in China less than 5 years ago, and it takes time for surgeons to become proficient in all aspects of the new procedure. Therefore, we recommend that future studies with larger sample sizes and longer follow-up periods are conducted to compare the healing rates of the two techniques.

In terms of anal function, the two groups were compared using the Wexner incontinence and FISI scores at 6 months postoperatively. The patients in the treatment group had significantly lower scores than those in the control group in both scales, indicating that the VAAFT method offers remarkable advantages when it comes to the protection of anal function and prevention of anal sphincter damage. The anus is an organ with many fine sensory functions. Damage to the anus can be lifelong, especially in young adults, resulting in a serious reduction in quality of life. Anal fistulas are particularly prevalent among young adults, thus the aim of protecting the anal sphincter is a common goal for patients and surgeons. In our findings, the VAAFT approach preserved anal function while providing excellent healing rates, almost comparable to traditional surgery.

There was no difference in the incidence of postoperative complications (e.g., urinary retention, anal edema, and bleeding) between the treatment and control groups, indicating that VAAFT is reliable in terms of surgical safety. Specifically, the closure of the internal opening using a stapler in VAAFT surgery causes no foreign-body sensation to patients, and the transverse internal-opening closure technique prevents edema of the anal edges because of its high position. Moreover, the clips are smaller than surgical sutures and therefore result in very low foreign-body irritation. At 1 month postoperatively, no residual clips were found in patients treated using a stapler, indicating that no patients suffered from long-term foreign-body irritation.

In the VAAFT group, the operating time was 15 minutes longer compared with the control group receiving traditional techniques, which correlates with surgical instrumentation and proficiency. Despite the VAAFT group needing a longer operating time, 15 minutes did not increase the overall difficulty of the procedure. However, patients in the VAAFT group showed significantly less intraoperative blood loss than the control group $(\mathrm{P}=0.007)$, possibly as a result of the minimally invasive nature of VAAFT, which involves fewer incisions with less damage, leading to less intraoperative blood loss. Although there was only a small amount of intraoperative blood loss in the conventional anal fistulotomy technique, a lesser degree of blood loss is one of the advantages of the VAAFT approach.

The wound healing time was significantly shorter in the VAAFT group than in the control group $(\mathrm{P}=0.001)$. As a minimally invasive procedure, the VAAFT technique requires a small incision site and involves closure of the internal opening; therefore, the duration of the procedure is also considerably reduced. This advantage is appealing to both surgeons and patients. Indeed, surgeons prefer outcomes with smaller wounds, easier wound management, and shorter dressing-change times.

Pain is a critical concern for patients who undergo surgery, especially anal and colorectal procedures. In this study, the NRS results of the VAAFT group on the first day, 1 week, and 1 month after the operation were $3.22 \pm 2.16,1.16 \pm 0.87$, and $0.54 \pm 0.51$, respectively, and those of the control group were $4.71 \pm 1.25,2.26 \pm 0.69$, and $0.66 \pm 0.71$, respectively. There were significant differences in the pain scores of the two groups recorded on the first day and 1 week after operation $(\mathrm{P}=0.001, \mathrm{P}=0.001$, respectively). In addition to respiration, pulse, body temperature, and blood pressure, pain is regarded as the fifth vital sign of life. Enhanced recovery after surgery (ERAS) (25) is a new concept in modern surgery, and its main goal is to relieve pain. The most effective way to reduce pain in surgery is minimal invasiveness, which reduces the incision range of tissue to achieve a small, or even no, incision. The VAAFT procedure is a minimally invasive surgery with a reduced incision size and smaller incision area. Moreover, VAAFT requires no incision 
of the anal sphincter, causing no sphincter spasm pain, and results in significantly less postoperative pain than the anal fistulotomy with seton placement procedure. The clear view of the anatomical structures of the anus provided by the fistuloscope is vital for identifying fistulas. The characteristics of the fistulas and other tissues under the fistuloscope are summarized as follows: (I) necrotic tissue: dark pink floc, floats in the solution, not fixed, falls off easily; (II) fistula tract wall: white elastic tissue, does not easily peel off or deform when touched; (III) muscle: soft tissue with straight red vertical bands; (IV) adipose tissue: loose connective tissue, reflective under the fistuloscope; and (V) fibrous tissue: white color, floats in the pipe, clamped with tension, does not easily fall off.

\section{Conclusions}

In summary, VAAFT offers numerous advantages including safety, effectiveness, minimal invasiveness, less pain, a shorter recovery time, and saving of the anal sphincter. Additionally, the internal-opening closure technique using a stapler in VAAFT can effectively treat the internal openings of anal fistulas, which is a promising clinical application.

\section{Acknowledgments}

Funding: This study was financially supported by the Beijing Municipal Science and Technology Commission [grant number Z181100001618004].

\section{Footnote}

Reporting Checklist: The authors have completed the CONSORT reporting checklist. Available at http://dx.doi. org/10.21037/atm-20-7154

Data Sharing Statement: Available at http://dx.doi. org/10.21037/atm-20-7154

Conflicts of Interest: All authors have completed the ICMJE uniform disclosure form (available at http://dx.doi. org/10.21037/atm-20-7154). The authors have no conflicts of interest to declare.

Ethical Statement: The authors are accountable for all aspects of the work in ensuring that questions related to the accuracy or integrity of any part of the work are appropriately investigated and resolved. The study protocol was conducted in accordance with the Declaration of Helsinki (as revised in 2013), and approved by the Research Ethics Committee of our hospital (2019ELLHA-001-01). Written informed consent was obtained from all patients prior to surgery.

Open Access Statement: This is an Open Access article distributed in accordance with the Creative Commons Attribution-NonCommercial-NoDerivs 4.0 International License (CC BY-NC-ND 4.0), which permits the noncommercial replication and distribution of the article with the strict proviso that no changes or edits are made and the original work is properly cited (including links to both the formal publication through the relevant DOI and the license). See: https://creativecommons.org/licenses/by-nc-nd/4.0/.

\section{References}

1. Visscher AP, Schuur D, Roos R, et al. Long-term followup after surgery for simple and complex cryptoglandular fistulas: fecal incontinence and impact on quality of life. Dis Colon Rectum 2015;58:533-9.

2. Meinero P, Mori L. Video-assisted anal fistula treatment (VAAFT): a novel sphincter-saving procedure for treating complex anal fistulas. Tech Coloproctol 2011;15:417-22.

3. Agha R, Abdall-Razak A, Crossley E, et al. STROCSS 2019 Guideline: Strengthening the reporting of cohort studies in surgery. Int J Surg 2019;72:156-65.

4. Singh RR, Nussbaum JS, Kumta NA. Endoscopic management of perforations, leaks and fistulas. Transl Gastroenterol Hepatol 2018;3:85.

5. Garg P. Is fistulotomy still the gold standard in present era and is it highly underutilized?: An audit of 675 operated cases. Int J Surg 2018;56:26-30.

6. Soltani A, Kaiser AM. Endorectal advancement flap for cryptoglandular or Crohn's fistula-in-ano. Dis Colon Rectum 2010;53:486-95.

7. Johnson EK, Gaw JU, Armstrong DN. Efficacy of anal fistula plug vs. fibrin glue in closure of anorectal fistulas. Dis Colon Rectum 2006;49:371-6.

8. Rojanasakul A. LIFT procedure: a simplified technique for fistula-in-ano. Tech Coloproctol 2009;13:237-40.

9. Isik O, Gulcu B, Ozturk E. Long-term Outcomes of Laser Ablation of Fistula Tract for Fistula-in-Ano: A Considerable Option in Sphincter Preservation. Dis Colon Rectum 2020;63:831-6.

10. Herreros MD, Garcia-Arranz M, Guadalajara H, et al. Autologous expanded adipose-derived stem cells for the 
treatment of complex cryptoglandular perianal fistulas: a phase III randomized clinical trial (FATT 1: fistula Advanced Therapy Trial 1) and long-term evaluation. Dis Colon Rectum 2012;55:762-72.

11. Meinero P, Mori L, Gasloli G. Video-assisted anal fistula treatment: a new concept of treating anal fistulas. Dis Colon Rectum 2014;57:354-9.

12. Adegbola SO, Sahnan K, Pellino G, et al. Short-term efficacy and safety of three novel sphincter-sparing techniques for anal fistulae: a systematic review. Tech Coloproctol 2017;21:775-82.

13. Rios HP, Goulart A, Rolanda C, et al. Enterocutaneous fistula: a novel video-assisted approach. Wideochir Inne Tech Maloinwazyjne 2017;12:297-300.

14. Giarratano G, Shalaby M, Toscana C, et al. Video-assisted anal fistula treatment for complex anal fistula: a long-term follow-up study. Colorectal Dis 2020;22:939-44.

15. Regusci L, Fasolini F, Meinero P, et al. Video-Assisted Anal Fistula Treatment (VAAFT) for complex anorectal fistula: efficacy and risk factors for failure at 3-year followup. Tech Coloproctol 2020;24:741-6.

16. Liu H, Tang X, Chang Y, et al. Comparison of surgical outcomes between video-assisted anal fistula treatment and fistulotomy plus seton for complex anal fistula: A propensity score matching analysis - Retrospective cohort study. Int J Surg 2020;75:99-104.

17. Cheung FY, Appleton ND, Rout S, et al. Videoassisted anal fistula treatment: a high volume unit initial experience. Ann R Coll Surg Engl 2018;100:37-41.

18. Pini Prato A, Zanaboni C, Mosconi M, et al. Preliminary

Cite this article as: Zhang Y, Li F, Zhao T, Cao F, Zheng Y, Li A. Efficacy of video-assisted anal fistula treatment combined with closure of the internal opening using a stapler for Parks II anal fistula. Ann Transl Med 2020;8(22):1517. doi: 10.21037/atm20-7154 results of video-assisted anal fistula treatment (VAAFT) in children. Tech Coloproctol 2016;20:279-85.

19. Liaqat N, Iqbal A, Dar SH, et al. Video Assisted Anal Fistula Treatment in a Child with Perianal Fistula. APSP J Case Rep 2016;7:3.

20. Adegbola SO, Sahnan K, Tozer PJ, et al. Symptom Amelioration in Crohn's Perianal Fistulas Using VideoAssisted Anal Fistula Treatment (VAAFT). J Crohns Colitis 2018;12:1067-72.

21. Schwandner O. Video-assisted anal fistula treatment (VAAFT) combined with advancement flap repair in Crohn's disease. Tech Coloproctol 2013;17:221-5.

22. Zarin M, Khan MI, Ahmad M, et al. VAAFT: Video Assisted Anal Fistula Treatment; Bringing revolution in Fistula treatment. Pak J Med Sci 2015;31:1233-5.

23. Emile SH, Elfeki H, Shalaby M, et al. A Systematic review and meta-analysis of the efficacy and safety of videoassisted anal fistula treatment (VAAFT). Surg Endosc 2018;32:2084-93.

24. Amato A, Bottini C, De Nardi P, et al. Evaluation and management of perianal abscess and anal fistula: a consensus statement developed by the Italian Society of Colorectal Surgery (SICCR). Tech Coloproctol 2015;19:595-606.

25. Slim K, Theissen A. Enhanced recovery after elective surgery. A revolution that reduces post-operative morbidity and mortality. J Visc Surg 2020;S1878-7886(20)30189-2.

(English Language Editor: A. Kassem) 